\title{
Active films based on thermoplastic corn starch and chitosan oligomer for food packaging applications
}

\author{
Luciana A. Castillo ${ }^{\mathrm{a}, \mathrm{b}}$, Sonia Farenzena ${ }^{\mathrm{c}}$, Esteban Pintos ${ }^{\mathrm{a}}$, María Susana Rodríguez ${ }^{\mathrm{c}}$, \\ Marcelo A. Villar ${ }^{\mathrm{a}, \mathrm{b}}$, María Alejandra García ${ }^{\mathrm{d}}$, Olivia V. López ${ }^{\mathrm{a}, *}$ \\ a Planta Piloto de Ingeniería Química (UNS-CONICET), Camino La Carrindanga Km 7, 8000 Bahía Blanca, Argentina \\ b Departamento de Ingeniería Química (UNS), Av. Alem 1253, 8000 Bahía Blanca, Argentina \\ c Departamento de Química (UNS), Av. Alem 1253, 8000 Bahía Blanca, Argentina \\ d Centro de Investigación y Desarrollo en Criotecnología de Alimentos (UNLP-CONICET), 47 y 116 s/n, 1900 La Plata, Argentina
}

\section{A R T I C L E I N F O}

\section{Keywords:}

Active biodegradable films

Thermoplastic corn starch

Chitosan oligomer

Diffusion assays

Antimicrobial capacity

\begin{abstract}
A B S T R A C T
Antimicrobial biodegradable films based on thermoplastic corn starch (TPS) and chitosan oligomers (CO) were obtained in order to develop a package prototype for perishable food products. Active films were fabricated thermo-compressing a sandwiched structure constituted by a thin layer of an oligomers enriched solution between two TPS films, previously made by melt-mixing and hot-pressing. Regarding enriched solution, it was obtained by dissolving oligomers in diluted acetic acid. Final material was characterized, determining its physical and optical properties, as well as, studying its microstructure. By diffusion assays it was demonstrated the capability of CO to migrate from the active film towards the aqueous simulant media. Moreover, oligomers were able to diffuse from the matrix, regardless the aqueous medium acidity. Experimental data of diffusion assays were fitted using a mathematical model, estimating diffusion coefficients at three studied pH values $(3,5$, and 7$)$. Active film was used to obtain sachets to package perishable foods such as strawberries, ricotta, and flavored breads, which were stored for 7 days under controlled conditions. Antimicrobial capacity of active sachets was corroborated through molds and yeast counts in the stored food products. Additionally, it was demonstrated that $\mathrm{CO}$ incorporation to the packaging material resulted in a more efficient way to inhibit microbial development than the spraying technique.
\end{abstract}

\section{Introduction}

Perishable foods are naturally susceptible to spoilage produced by microorganism attacks, for this reason, some method of protection is necessary to avoid product deterioration during the entire supply chain. Hence, antimicrobial agents are usually used to extent food shelf-life. Nowadays, the use of natural compounds to preserve food products is exponentially growing due to the awareness about synthetic chemical additives from consumers. In this regard, Lucera, Costa, Conte, and Del Nobile (2012) reported the use of essential oils derived from plants (e.g., basil, thyme, oregano, cinnamon, clove, and rosemary), animal enzymes (e.g., lysozyme, lactoferrin), microbial bacteriocins (nisin, natamycin), organic acids (e.g., sorbic, propionic, citric acid) and biopolymers (chitosan). Several methodologies were employed to protect foods by using antimicrobials: they can be incorporated during food processing, coated on products surface or included into the packaging material. Among direct methods, dipping, impregnation, coating, and spraying are the different ways of application of active agents at industrial scale because of its simplicity, low cost, and versatility (López, Giannuzzi, Zaritzky, \& García, 2013). However, one of its disadvantages is the efficiency loss of the agent functionality, which forces the use of relatively concentrated active solutions. In this sense, the main drawbacks associated to spraying technique are the occurrence of secondary reactions between active agents with food components, decreasing the method effectiveness. Some food substances, such as proteins, proteases, lipids, salts, and metal ions, can interfere in the interaction of antimicrobials by reacting directly with them or by interacting with their target microorganism (Juneja, Dwivedi, \& Yan, 2012; Touch, Hayakawa, \& Commins, 2009). Besides, antimicrobial compounds could migrate quickly into the bulk product, leaving its surface unprotected (Pranoto, Rakshit, \& Salokhe, 2005). Therefore, the development of active packaging materials containing antimicrobials could be an interesting alternative to optimize the agent activity and efficiency. It is important to highlight that antimicrobial agents must gradually diffuse through a polymeric matrix towards the food product. This gradual release allows maintaining an effective concentration of

\footnotetext{
* Corresponding author.

E-mail address: olivialopez@plapiqui.edu.ar (O.V. López).
} 
antimicrobial agent on food surface for longer periods of time, being an advantage over the spraying technique (Appendini \& Hotchkiss, 2002). Active agent diffusion from the packaging material is affected by several factors. Within this context, Kuorwel, Cran, Sonneveld, Miltz, and Bigger (2013) claimed that this phenomenon depends on: i) film preparation method, ii) intrinsic agent characteristics, iii) agent-polymer chemical interactions, iv) changes in packaging film induced by the agent presence, v) inherent matrix characteristics, vi) composition, water activity and $\mathrm{pH}$ of the food, and vii) storage conditions.

Nowadays, there is a continuously growing interest in the use of biodegradable materials to develop food active packaging, not only for ecological reasons but also from a practical point of view (Srinivasa \& Tharanathan, 2007). In this sense, starch is a promising alternative because of its low cost, world availability, and functionality. Starch processing under high temperature and shear stress, in the presence of plasticizers, allows obtaining thermoplastic starch (TPS), (Castillo et al., 2013). Several research papers of starch active films for food packaging can be found in the literature. For example, potassium sorbate incorporation into tapioca starch-glycerol edible films prevented external $Z$. bailii contamination and controlled yeast growth in an acidified $(\mathrm{pH} 4.5)$ high water activity (aw $=0.980)$ semisolid product (Flores, Haedo, Campos, \& Gerschenson, 2007). Besides, Barzegar, Azizi, Barzegar, and Hamidi-Esfahani (2014) demonstrated that starch-clay nanocomposite films which contained potassium sorbate also had antimicrobial property against Aspergillus niger. On the other hand, chitosan and lauric acid were added to starch films at different proportions evidencing an inhibitory effect on Bacillus subtilis and Eschericha coli growth (Salleh, Muhammad, \& Pahlawi, 2014). Glycerol plasticized potato starch films containing bioactive proteins (lactoferrin and/or lysozyme) were developed by Moreno, Atarés, \& Chiralt, (2015). In this work the authors stressed that even though proteins incorporation affected films structural and physical properties, antimicrobial action against Escherichia coli and coliforms was observed. Besides, essential oils such as linalool, carvacrol, or thymol have been incorporated to starch based materials as active compounds (Cano, Cháfer, Chiralt, \& González-Martínez, 2015; Kuorwel, Cran, Sonneveld, Miltz, \& Bigger, 2011; Kuorwel et al., 2013; Mehdizadeh, Tajik, Razavi Rohani, \& Oromiehie, 2012). Particularly, Kuorwel et al. (2013) studied the migration of these antimicrobial agents from starch-based film samples into isooctane as a fatty-food simulant and demonstrated their high release efficiencies, allowing to extend food shelf-life and to reduce microbial contamination.

Another promising candidate as antimicrobial agent of starch packaging systems is chitosan (van den Broek, Knoop, Kappen, \& Boeriu, 2015). It is a natural carbohydrate polymer obtained by the deacetylation of chitin, a major component of crustacean's shells. Chitosan presents antimicrobial and antifungal activity against a wide range of microorganisms and it has been extensively studied as food preservative (Corrales, Fernández, \& Han, 2014; Srinivasa \& Tharanathan, 2007). The biological activity of chitosan depends on its molecular weight, degree of deacetylation and derivatization, such as, degree of substitution, length and position of a substitute in the glucosamine units of chitosan, $\mathrm{pH}$ of the chitosan solution and the target organisms (Lucera et al., 2012; No, Meyers, Prinyawiwatkul, \& Xu, 2007). Several models suggested that the antimicrobial activity of chitosan is a result from its cationic nature (Goy, de Britto, \& Assis, 2009; Rabea, Badawy, Stevens, Smagghe, \& Steurbaut, 2003). The electrostatic interaction between positively charged $R \mathrm{~N}$ $\left(\mathrm{CH}_{3}\right)^{3+}$ sites and negatively charged microbial cell membranes, is predicted to be responsible for cellular lysis and assumed as the main antimicrobial mechanism (Goy, Morais, \& Assis, 2016; Tripathi, Mehrotra, \& Dutta, 2008). In accordance to Romainor, Chin, Pang, and Bilung, (2014), chitosan antimicrobial effect could be increased by reducing its molecular size. In this sense, the small size of chitosan rendered them with unique physicochemical properties such as large surface area (providing more cationic sites) and high reactivity and thus, could potentially enhance the charge interaction on the microbial surface and lead to a greater antimicrobial effect (Zhang, Pornpattananangkul, Hu, \& Huang, 2010). Hussain, Singh, and Chittenden (2012) stressed that antifungal activity increases by decreasing the degree of polymerization of chitosan oligomers, which is divergent to literature data. Within this context, the use of low molecular weight chitosan as antimicrobial agent of starch packaging would be an encouraging strategy not only to increase its antimicrobial effect but also to favor its diffusion ability. The use of chitosan oligomers is doubly advantageous due to their inherent functionality as antimicrobial agent, as well as, to their natural source. Chitosan and its derivatives are obtained from fisheries waste, giving added value to these high-volume and low-cost residues (Brück, Slater, \& Carney, 2010). Particularly, oligochitosan is obtained from shrimp shells wastes which are constituted by chitin, the value-added precursor of chitosan. Because chitin is an easily accessible resource, the full exploitation/bioconversion of this polysaccharide is of great interest for both, the industrial and the academic field. Thus, oligochitosan incorporation to TPS matrix would lead to a biodegradable material with an additional functionality. It is important to mention that the use of chitosan derivatives is a less investigated topic to obtain active packaging.

The aim of this work was to obtain a package prototype for perishable food products from active films based on thermoplastic corn starch (TPS) and chitosan oligomers (CO) which could extend food shelf-life. This active package would be considered as an alternative to the conventional spraying method.

\section{Materials and methods}

\subsection{Materials}

Native corn starch was provided by Misky-Arcor (Tucumán, Argentina) with an amylose content of $23.9 \pm 0.7 \%$ (López, García, \& Zaritzky, 2008). Chitosan oligomer (CO) was obtained by oxidative degradation of chitosan, assisted by microwave radiation, using a modification of the methodology proposed by Shao, Yang, \& Zhong, (2003). The chitosan used as raw material $\left(\mathrm{M}_{\mathrm{W}}=468,200 \mathrm{~g} \mathrm{~mol}^{-1}\right)$ was obtained from chitin of shrimp shells wastes (Pleoticus mülleri) through the method proposed by Zuñiga, Debbaudt, Albertengo, and Rodríguez (2010). Shrimp shells are a fraction of the wastes resulting from the fishing industry. The yield of the CO synthesis reaction was determined following the methodology proposed by Shao et al. (2003). After irradiation had been stopped, the product was cooled and filtered under reduced pressure. The obtained residue and the filter paper were dried to constant weight in an infrared dryer. By deducting the filter paper weight, the mass of the unreacted chitosan was calculated, determining the amount of water-soluble oligomers.

\subsection{Characterization of chitosan oligomer}

The number average molecular weight and degree of polymerization of chitosan oligomer (CO) were determined by the method of end group analysis (Shao et al., 2003). Morphological characterization was examined by Scanning Electron Microscopy (SEM) using a JEOL JSM$35 \mathrm{CF}$ microscope with a secondary electron detector.

Structural characterization was carried out by Fourier Transform Infrared Spectroscopy (FTIR) using a Thermo Nicolet Nexus spectrophotometer. Samples were prepared by milling and mixing $\mathrm{CO}$ with $\mathrm{KBr}$ (Sigma-Aldrich, 99\%) at $1 \% \mathrm{w} / \mathrm{w}$. The mixture was pressed and a transparent sample was achieved. Spectra were obtained from 100 accumulated scans at $4 \mathrm{~cm}^{-1}$ resolution in the range $4000-400 \mathrm{~cm}^{-1}$.

Thermal properties were determined by Differential Scanning Calorimetry (DSC) and Thermogravimetric Analysis (TGA). For DSC runs, a Perkin Elmer Pyris I calorimeter was used. Approximately $10 \mathrm{mg}$ of $\mathrm{CO}$ were weighted in hermetic pans in order to avoid water loss during the assays. An empty hermetic pan was used as reference. 
Samples were heated from 20 to $250{ }^{\circ} \mathrm{C}$ at $10{ }^{\circ} \mathrm{C} / \mathrm{min}$, under nitrogen atmosphere. From thermograms, the following melting parameters were obtained: onset $\left(\mathrm{T}_{\mathrm{o}}\right)$ and peak $\left(\mathrm{T}_{\mathrm{m}}\right)$ temperatures, and enthalpy $\left(\Delta \mathrm{H}_{\mathrm{m}}\right)$. Thermal degradation was carried out in a thermogravimetric balance TA Instrument Discovery Series. Samples were heated from 30 to $700{ }^{\circ} \mathrm{C}$ at $10^{\circ} \mathrm{C} / \mathrm{min}$, under air atmosphere. Curves of loss weight as function of temperature were recorded.

Since the characteristics and properties of the derivatives are strongly dependent on the original chitosan source, this polysaccharide was also analyzed by SEM, DSC and TGA using the same conditions employed for $\mathrm{CO}$ characterization.

\subsection{Active films}

In order to prepare active films, chitosan oligomer (CO) was dissolved in $0.1 \% \mathrm{v} / \mathrm{v}$ acetic acid, obtaining an enriched solution with a concentration of $0.3 \mathrm{~g} / \mathrm{mL}$. In parallel, thermoplastic starch (TPS) films were obtained using the same component proportions and processing conditions reported in a previous work (Castillo et al., 2013). The CO enriched solution was spread onto a TPS film, obtaining a thin and homogeneous coating. Then, another TPS film was put over the CO covered film and the system was thermo-compressed using a hydraulic press at $90{ }^{\circ} \mathrm{C}$ during $5 \mathrm{~min}$. A schematic representation of the active system is shown in Fig. 1a. Prior to the characterization and diffusion assays, the films were conditioned at $25^{\circ} \mathrm{C}$ and $50 \% \mathrm{RH}$ until a constant weight was reached. The final $\mathrm{CO}$ concentration in the films was $0.12 \mathrm{~g}$ per gram of TPS.

\subsection{Characterization of active films}

Thickness of films was determined at different randomized locations of the specimens and the reported value corresponds to the mean thickness. Besides, films density was calculated from mass per volume unit.

Films microstructure was studied through Scanning Electron Microscopy (SEM) in a JEOL JSM-35 CF microscope with a secondary electron detector, using an accelerating voltage of $10 \mathrm{kV}$. Films were

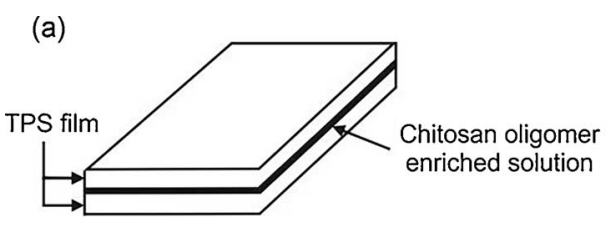

(b)

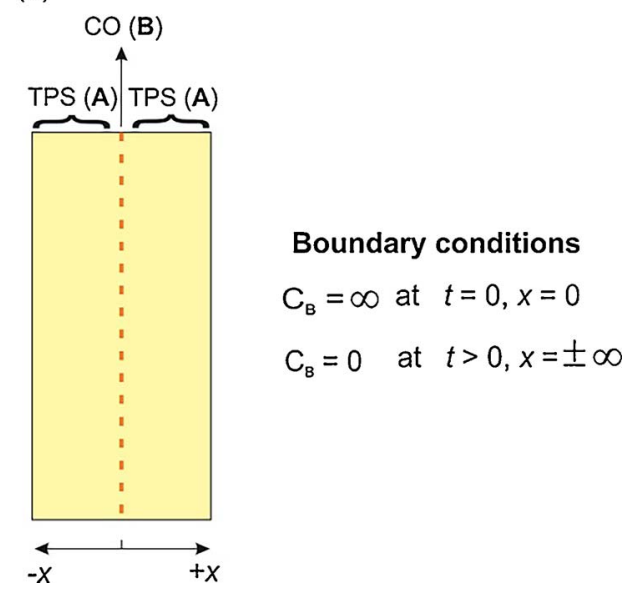

Fig. 1. Schematic representation of: a) active film based on thermoplastic corn starch (TPS) and chitosan oligomer and b) the active system and boundary conditions. previously cryo-fractured by immersion in liquid nitrogen, mounted on bronze stubs, and coated with a gold layer.

Color parameters were determined using a Hunterlab UltraScan XE colorimeter in the transmittance mode. Chromaticity $\left(a^{*}\right.$ and $\left.b^{*}\right)$, as well as, luminosity $\left(L^{*}\right)$ values were obtained according to the CIELAB scale, testing at least ten randomly selected positions on the films by triplicate. Parameters corresponding to the white background $\left(L^{*}=94.42\right.$, $a^{*}=-0.67$, and $\left.b^{*}=0.61\right)$ were considered as standard values. Moreover, yellowness index (YI) was determined using the formula reported by Pathare, Opara, and Al-Said (2013):

$Y I=\frac{142.86 b^{*}}{L^{*}}$

Ultraviolet light barrier property and transparency were determined using UV-vis Spectroscopy. Films were cut into rectangles and placed into a quartz cell to record the absorbance spectrum $(200-800 \mathrm{~nm})$ in a PG Instruments T60 UV/vis spectrophotometer. UV barrier capacity was quantified through the area under the peak located between 270 and $300 \mathrm{~nm}$. Film transparency was estimated by using ASTM 148 D1746-97 method and according to the procedure reported by Alvarado-González et al. (2012). Both optical properties were determined by triplicate.

\subsection{Diffusion assays}

Diffusion of chitosan oligomer (CO) from the active film was evaluated by immersing pre-weighed specimens $(\sim 60 \mathrm{mg})$ into distilled water ( $3 \mathrm{~mL}$ ) as simulant aqueous medium at $\mathrm{pH} 7$. In order to evaluate the effect of the $\mathrm{pH}$ medium on $\mathrm{CO}$ diffusion, two additional simulant solutions were prepared by acidifying distilled water at $\mathrm{pH} 3$ and 5 . Diffusion assays were performed at room temperature $\left(20 \pm 2{ }^{\circ} \mathrm{C}\right)$. Aliquots were taken at selected times $(30,60,120,180$, and $300 \mathrm{~min})$ and the oligomer concentration was quantified spectrophotometrically, following the D-glucosamine procedure described by Shao et al. (2003). This method was carried out using a Spectronic 21D equipment and reported values correspond to the average of, at least, two determinations for each assayed time and $\mathrm{pH}$.

Mathematical model: experimental data of diffusion assays were adjusted using a mathematical model based on the Fick's second law (Eq. (2)).

$\frac{\partial C}{\partial t}=D \nabla^{2} C$

where $C$ is the CO concentration in the solution, $t$ is the diffusion time, and $D$ is the diffusion coefficient.

This model assumes that the diffusion coefficient $(D)$ is concentration and position independent. As it was explained in detail before, the studied material was constituted by a very thin layer of homogeneous B material (CO enriched solution) located between two films of A material (TPS). Fig. 1b shows a schematic representation of this sandwiched system. Considering that thickness of $\mathbf{B}$ is negligible compared to the thickness of A, B self-diffuses through A films and the "thin film solution" approximation can be applied. Denoting $x$ as the axis where the thickness was measured and neglecting diffusion in other directions, Eq. (2) is simplified to:

$\frac{\partial C_{B}}{\partial t}=D \frac{\partial^{2} C_{B}}{\partial x^{2}}$

The solution to Eq. (3) is as follows:

$C(x, t)=\frac{N}{\sqrt{4 \pi D t}} e^{\left(-\frac{x^{2}}{4 D t}\right)}$

The expression of Eq. (4) is symmetrical respect to $x=0$ and it tends to 0 as $x$ approaches either $+\infty$ or $-\infty$ for any $t>0$. Meanwhile, for $t=0$ it only exists at $x=0$ where $\mathrm{C}_{\mathrm{B}}$ becomes $\infty$. This last value $\left(C_{B}=\infty\right)$ is hypothetic and corresponds to $\mathrm{CO}$ concentration at 
the initial time where B material (in pure state) is an infinite source of $\mathrm{CO}$. These boundary conditions for the studied system are included in Fig. 1 b.

Taking into account the aforementioned conditions and considerations, Eq. (4) can be analytically integrated to obtain the following expression:

$\int_{-x}^{x} C(x, t)=\frac{1}{2} N \operatorname{erf}\left(\frac{x}{2 \sqrt{D t}}\right)$

where $N$ is the initial quantity of CO in the thin B layer and erf is the error function.

Assuming a given value of $D$, the CO amount in TPS films was calculated from Eq. (5), considering the chosen boundary conditions. Then, the CO amount which diffused to the simulant medium was determined by the numerical difference between $N$ and the CO amount in TPS film after a certain time. It is important to highlight that it was considered that TPS swelling and dissolution were negligible, as well as, the interfacial resistance between TPS films and the simulant. Thus, taking into account that the thickness of the studied system was $280 \mu \mathrm{m}$ (thickness of each TPS layer $=140 \mu \mathrm{m}$ ) and considering $N=1$, the diffusion coefficients were estimated for the three $\mathrm{pH}$ values from the following expression:

$N_{D}=\left[1-\left[\operatorname{erf}\left(\frac{-x}{2 \sqrt{t D}}\right)-\operatorname{erf}\left(\frac{x}{2 \sqrt{t D}}\right)\right]\right] \times 100$

where $x$ is expressed in centimeters and $t$ in seconds. Finally, experimental data were adjusted to the model using the least square method.

\subsection{Antimicrobial capacity}

Microbiological analysis were performed to evaluate comparatively the use of active packages against the spraying method by an enriched solution, considering in both cases chitosan oligomer as the antimicrobial agent. For these studies, fruit, dairy, and bakery products were chosen as reference perishable foods. Fresh whole strawberries were selected considering fruits with similar sizes, maturity grades, and no visible physical damages. It is important to mention that no sanitization pre-treatments were applied on strawberries to reduce their initial microbial charge. Whole moldy ricotta $(3 \mathrm{~kg})$ was used as dairy derivative from cow milk which was carefully fractioned to reduce sample size $(\sim 20 \mathrm{~g})$. Mignon breads with cheese flavor were bought as unpacked products.

Spraying solution was prepared by dissolving CO in $0.1 \% \mathrm{v} / \mathrm{v}$ acetic acid and adding distilled water, obtaining a final concentration of $0.05 \mathrm{mg} / \mathrm{mL}$.

Sachet type packages based on the active film were obtained by thermosealing, adapting the methodology described by López, Castillo, García, Villar, and Barbosa (2015). Sachets were conditioned at $25{ }^{\circ} \mathrm{C}$ and $50 \% \mathrm{RH}$ up to equilibrium state and then, they were individually weighted to calculate the $\mathrm{CO}$ amount contained in each active package. Based on this oligomer quantity, the volume of the enriched solution was established, assuring that the $\mathrm{CO}$ amount involved in both methodologies was the same.

Samples preparation: in the case of strawberries, they were assayed without removing fruits peduncles. Meanwhile, portions of ricotta $(20 \mathrm{~g})$ and mignon breads $(\sim 30 \mathrm{~g})$ were fractioned under aseptic conditions. Individual fruits and mignons, as well as, ricotta portions were packed inside the active sachets, assuring a direct contact between the product and the packaging material. These samples were named as PS (packed strawberry), PR (packed ricotta), and PB (packed bread). To evaluate the effect of the spraying method, several specimens of each food product were coated with the $\mathrm{CO}$ enriched solution by atomization. These samples were named as SS (sprayed strawberry), SR (sprayed ricotta), and SB (sprayed bread). All samples were stored at $25{ }^{\circ} \mathrm{C}$ and $50 \%$ HR during 7 days. In parallel, control samples of each food were conserved under the same conditions. These specimens were named as CS (control strawberry), CR (control ricotta), and CB (control bread). All the assays were performed by triplicate.

Microbiological assays: at the end of the storage time, $10 \mathrm{~g}$ of each specimen corresponding to the assayed foods were homogenized in a Stomacher Seward Model 400 with $90 \mathrm{~mL}$ of sterile peptone water $(1 \%$ $\mathrm{w} / \mathrm{v}$ ). Aliquots of $1 \mathrm{~mL}$ of each homogenate were seeded by triplicate in $3 \mathrm{M}^{\mathrm{TM}}$ Petrifilm ${ }^{\mathrm{TM}}$ Count Plates for molds and yeasts. All plates were incubated at $37{ }^{\circ} \mathrm{C}$ during 7 days. Then, total molds and yeasts count was carried out and reported values were expressed as colony-forming units (CFU) per food gram.

\subsection{Statistical analysis}

Analysis of variance (ANOVA) was used to compare mean differences of films properties, as well as, the results derived from diffusion and antimicrobial capacity assays. Besides, comparison of mean values was performed by Fisher's least significant difference test conducted at a significance level $p=0.05$.

\section{Results and discussion}

\subsection{Characterization of chitosan oligomers}

The study of oligochitosan structural characteristics is relevant since they determine potential applications of this low molecular weight polysaccharide. Chitosan oxidative degradation using microwave radiation allowed to achieve an oligomer yield of $41.0 \pm 0.3 \%$. This value is in accordance with the percentages reported by Shao et al. (2003) for oligomers prepared by oxidative degradation of chitosan with neutral hydrogen peroxide under microwave irradiation.

The number average molecular weight of the synthetized CO was $290 \mathrm{~g} \mathrm{~mol}^{-1}$. This physical property is strongly affected by reaction conditions mainly oxidizing agent concentration, oxidation and irradiation time, and amount of chitosan, among others. Taking into account this dependence, differences between the obtained molecular weight with those reported in the literature are based on the fact that oligomers synthesis was performed under dissimilar operating parameters (Hussain et al., 2012). Considering that molecular weight of glucosamine repeating units is $161 \mathrm{~g} \mathrm{~mol}^{-1}$, the degree of polymerization of $\mathrm{CO}$ was around 2. A reduction in chitosan molecular weight supposes an increment in its antimicrobial capacity as a consequence of a higher surface area, which provides more cationic sites (Romainor et al., 2014). These positive charges interact with negatively charged microbial cell membranes, leading to their lysing (Goy et al., 2016; Tripathi et al., 2008). Besides, the low size of chitosan derivatives would allow a high mobility and diffusion ability in several media. This additional functionality potentiates the inherent antimicrobial capacity of chitosan derivatives, broadening their application field.

Fig. 2a includes SEM micrographs of the chitosan before and after oxidative degradation. As it can be observed, before treatment chitosan shows a characteristic flaky-like structure, described by Mohamed, Elella, \& Sabaa, (2015). When chitosan was exposed to the oxidative degradation, polysaccharide morphology changed significantly. SEM micrographs of chitosan after degradation evidence the presence of spherical shaped units, with smooth surfaces and uniform sizes. Similar observations were stressed by Khanmohammadi, Elmizadeh, and Ghasemi (2015), regardless the synthesis method of chitosan oligomers was spontaneous emulsification instead of oxidative degradation assisted by microwave radiation. The notable changes in chitosan structure are in accordance with the aforementioned low molecular weight and degree of polymerization of the synthetized CO. From SEM micrographs, it was determined that the mean diameter of $\mathrm{CO}$ resulted of $1.5 \pm 0.2 \mu \mathrm{m}$, measuring at least one hundred spherical units. In Fig. 2a, it is observed that chitosan partially conserves its initial morphology (flaky-like structure) in accordance with the efficiency of the 

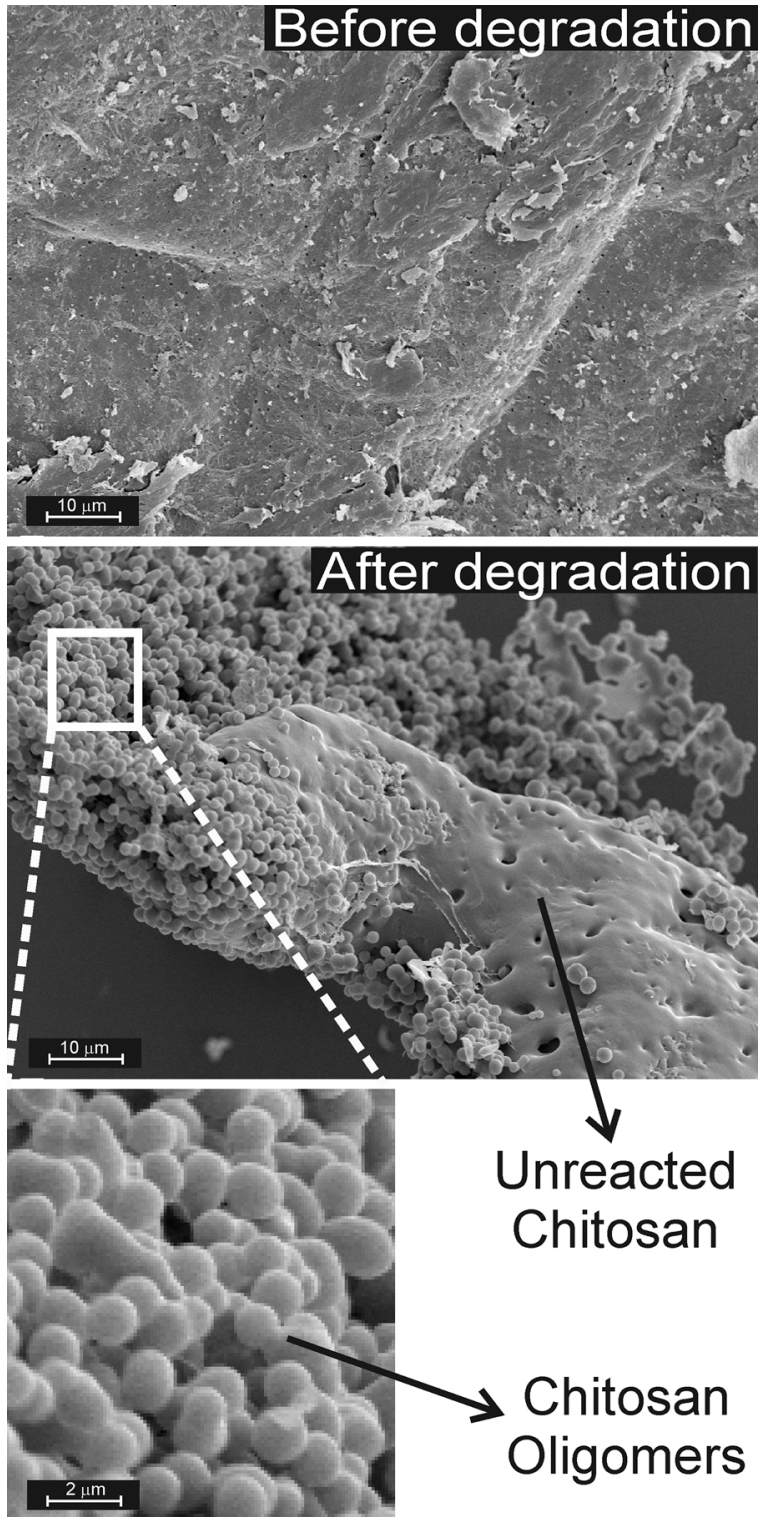

Fig. 2. a) SEM micrographs of chitosan before and after oxidative degradation, b) FTIR spectra and c) TGA curves of chitosan and chitosan oligomer.

oxidative degradation, quantified through the reaction yield (41\%).

FTIR spectroscopy was used to assess the polymer chemical groups in the molecular structure. Fig. 2b shows the spectra of the initial chitosan and its derivative, including the main absorption bands. Regarding chitosan spectrum, signal at $3735 \mathrm{~cm}^{-1}$ is assigned to nonhydrogen bonded $\mathrm{OH}$ groups (Rawat \& Singh, 2015). The broad peak at $3450 \mathrm{~cm}^{-1}$ is related to stretching vibration of $\mathrm{O}-\mathrm{H}$ and $\mathrm{N}-\mathrm{H}$ bonds. Symmetric stretching vibration of $\mathrm{C}-\mathrm{H}$ in alkyl group is detected at 2918 and $2881 \mathrm{~cm}^{-1}$. Besides, stretching vibrations of $\mathrm{C}-\mathrm{O}$ bond of the remaining $\mathrm{N}$-acetyl group are distinguished at 1653 and $1323 \mathrm{~cm}^{-1}$. The band corresponding to $\mathrm{N}-\mathrm{H}$ bending vibration of the amino groups appears at $1593 \mathrm{~cm}^{-1}$. Peaks at 1379 and $1419 \mathrm{~cm}^{-1}$ are assigned to the $\mathrm{CH}_{3}$ symmetrical deformation mode. Bands corresponding to $\mathrm{C}-\mathrm{O}-\mathrm{C}$ bending vibration in chitosan saccharide repeating unit can be found at 1151 and $895 \mathrm{~cm}^{-1}$. Absorption band at $1254 \mathrm{~cm}^{-1}$ is attributed to the amine or amide functional groups. The peaks located at 1084 and $1031 \mathrm{~cm}^{-1}$ are assigned to $\mathrm{C}-\mathrm{OH}$ stretching vibration. A similar spectrum was reported by Costa-Júnior, Barbosa-Stancioli, Mansur, Vasconcelos, and Mansur (2009); Mohamed et al. (2015) and Zainal, Hui, Hussein, Abdullah, and Hamadneh (2009). The band at
Table 1

Thermal properties associated to melting process of chitosan and its oligomer.

\begin{tabular}{llll}
\hline & $\begin{array}{l}\text { Onset temperature } \\
\left(\mathrm{T}_{\mathrm{o}},{ }^{\circ} \mathrm{C}\right)\end{array}$ & $\begin{array}{l}\text { Peak temperature } \\
\left(\mathrm{T}_{\mathrm{m}},{ }^{\circ} \mathrm{C}\right)\end{array}$ & $\begin{array}{l}\text { Enthalpy }\left(\Delta \mathrm{H}_{\mathrm{m}},\right. \\
\mathrm{J} / \mathrm{g})\end{array}$ \\
\hline $\begin{array}{llll}\text { Chitosan } \\
\text { Chitosan } \\
\text { oligomer }\end{array}$ & 117 & 147 & 295 \\
\hline
\end{tabular}

about $667 \mathrm{~cm}^{-1}$ is assigned to out-of-plane $-\mathrm{NH}$ deformation (Wu \& Zivanovic, 2008). FTIR spectrum of CO derivative demonstrated that oxidative degradation induced slight changes in the polysaccharide microstructure. In this sense, non-significant shifts in peaks location and the absence of some bands are detected. Chitosan signals observed at 1593,1254 , and $1084 \mathrm{~cm}^{-1}$ are not evidenced in the FTIR spectrum of the oligomers.

The knowledge of thermal properties of chitosan and its derivatives is relevant since they restrict the processing conditions of these polysaccharides. In this sense, results derived from thermal characterization allow setting their processing window. Table 1 includes thermal parameters related to the melting phenomenon, including the onset $\left(\mathrm{T}_{\mathrm{o}}\right)$ and peak $\left(\mathrm{T}_{\mathrm{m}}\right)$ temperatures, as well as, the enthalpy $\left(\Delta \mathrm{H}_{\mathrm{m}}\right)$ values. Chitosan and $\mathrm{CO}$ thermograms show an unique endothermic event. Regarding the chitosan thermal properties, these values are within the range of those reported in the literature (Nallamuthu, Devi, \& Khanum, 2015; Pillai \& Ray, 2012). It is well known that chitosan physicochemical characteristics such as molecular weight, polydispersion, and deacetylation degree, significantly influence on its thermal properties. Thus, it is probable to find a wide range of these parameters in the bibliography. Chitosan oxidative degradation induced a notable shift in $T_{o}$ and $T_{m}$ to lower values, as it was expected. In addition, a decrease in $\Delta \mathrm{H}_{\mathrm{m}}$ was also evidenced (Table 1). These results are the consequence of the polymeric chains shortening due to the chitosan degradation. The reduction of $\sim 50 \%$ in $\mathrm{T}_{\mathrm{m}}$ is completely in accordance with the low molecular weight and polymerization degree of this derivative. It is well known that melting properties of polymers depend on the molecular weight and polymerization degree, so the extent of the oligomers synthesis reaction will affect these physical properties.

Fig. 2c shows the temperature dependence of weight loss corresponding to chitosan and $\mathrm{CO}$, also including their derivative curves (DTGA). As it can be observed, chitosan and its derivative present three irreversible weight loss steps. The first one is associated to samples dehydration, registering for both polysaccharides a weight loss of $\sim 9 \%$. This thermal event corresponds to the loss of physically adsorbed and strongly hydrogen bonded water to chitosan (Corazzari et al., 2015). The second stage gives information about the degradation of chitosan and $\mathrm{CO}$ polymeric chains, being this one the most relevant to set the processing conditions. This weight loss is related to the depolymerization/decomposition of polymer chains through deacetylation and cleavage of glycosidic linkages (Moussout, Ahlafi, Aazza, \& Bourakhouadar, 2016). Thermal degradation associated to this event starts at $\sim 220^{\circ} \mathrm{C}$, presenting a maximum rate at $274^{\circ} \mathrm{C}$. These values are in good agreement with those reported by Ziegler-Borowska, Chełminiak, and Kaczmarek (2015). The last weight loss step corresponds to the thermal destruction of pyranose rings and the decomposition of the residual carbon (Corazzari et al., 2015; Marroquin, Rhee, \& Park, 2013). The effect of oxidative degradation on chitosan thermal stability is mainly observed by the shift to lower values of onset temperature corresponding to the depolymerization/decomposition of polymer chains. Moreover, instead of the unique peak associated to this thermal event in chitosan samples, a broad degradation range is detected in the CO curve. This behavior is based on the fact that oxidative degradation disrupted the chitosan structure by the disintegration of intra- and intermolecular interactions, decreasing the molecular weight of this polysaccharide (Zheng, Yin, Jiang, Xing, \& Pu, 2015). 


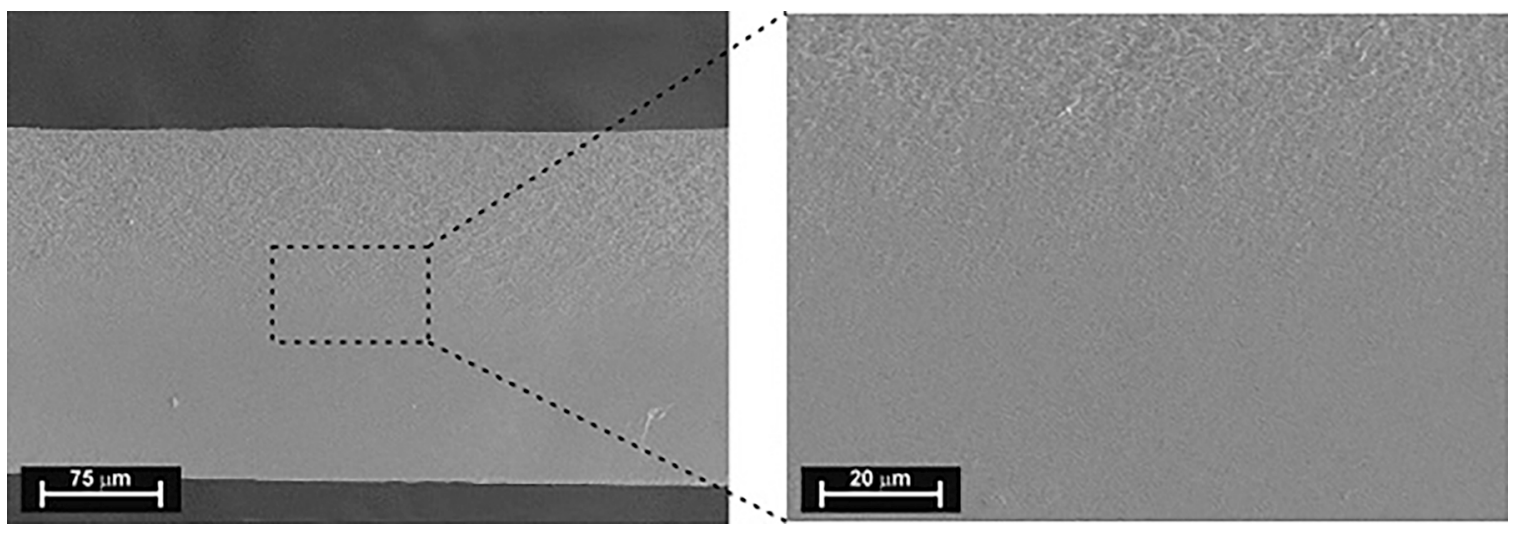

Fig. 3. SEM micrograph of active film based on thermoplastic corn starch (TPS) and chitosan oligomer.

\subsection{Characterization of active films}

The development of materials based on natural polysaccharides such as starch and chitosan derivatives are in accordance with the biotechnological criteria based on the renewable and biodegradable character of starch and chitosan.

Macroscopic evaluation of films based on TPS and CO revealed that they were flexible and easy to handle, having a homogeneous appearance. Moreover, they resulted in a soft-touch material, being translucent with a yellow-amber tone. Despite these film had a glossy finish, its texture was neither oily nor sticky. Active films presented a mean thickness of $280 \mu \mathrm{m}$ and a density of $1.428 \mathrm{~g} / \mathrm{cm}^{3}$.

Microstructure analysis of polymeric based materials allows the study of their surface homogeneity as well as the evaluation of the compatibility among different constituents. Fig. 3 shows SEM micrographs corresponding to active films at two different magnifications. Fracture surface resulted smooth, without pores, cracks nor channels induced by glycerol migration through the matrix. The last microscopic finding confirms the macroscopic evaluation of films texture respect to the lack of oiliness and stickiness when these materials were handled. SEM micrographs also revealed a brittle fracture, favored by the specimen immersion in liquid nitrogen, which allows evaluating film surface without any interference. No insoluble particles were detected within film structure, meaning that $\mathrm{CO}$ was completely dissolved in the acetic acid solution, before spreading onto the TPS film. Additionally, the lack of dispersed phases could be attributed to the good compatibility between chitosan derivative and the matrix, as well as, to the effectiveness of thermal processing. Thermoplastic starch films used to develop the active material had a thickness of around $190 \mu \mathrm{m}$, meanwhile the system containing CO was thicker $(\sim 280 \mu \mathrm{m})$. The absence of a defined interphase is an indicative of a complete integration of both TPS films and $\mathrm{CO}$ enriched solution during thermo-compression process. This observation is in accordance with the fact that the final thickness of the active system resulted lower than the expected value $(\sim 380 \mu \mathrm{m})$.

Among the final properties of materials tailored for food packaging, color characteristics are relevant since they condition the visual acceptability of the product by the consumer. Yellowness index of active films was $10.18 \pm 1.22$, being four times higher than the corresponding value of neat TPS film (Castillo et al., 2015). Color development in the active film is mainly associated to the non-enzymatic browning (Maillard reaction) which confers to this material a characteristic yellowish hue. This color alteration is promoted by processing conditions, as it was also reported by other authors (Guerrero, Beatty, Kerry, \& De La Caba, 2012; López et al., 2014). Products derived from the reactions involved in Maillard browning are Amadori compounds and insoluble polymers, referred as melanoidins (Leceta, Guerrero, \& de la Caba, 2013; Yasir, Sutton, Newberry, Andrews, \& Gerrard, 2007).

Another important property of food packaging materials is related to their capacity to absorb UV light. In this regard, spectrum corresponding to active films containing oligochitosan present an absorption peak located between 270 and $300 \mathrm{~nm}$, which evidences that CO did not affect UV absorption capacity of TPS films (Castillo et al., 2013). This characteristic is relevant to develop food packaging for products which are susceptible to oxidation process induced by this kind of radiation. The value of UV absorption capacity corresponding to active film resulted $47.40 \pm 3.50 \mathrm{AU} x \mathrm{~nm}$.

Concerning to transparency, films containing oligochitosan presented a value of $0.60 \pm 0.03 \mathrm{~mm}^{-1}$. This optical property does not present significant differences respect to TPS films $\left(0.52 \pm 0.14 \mathrm{~mm}^{-1}\right)$, (Castillo et al., 2015). The fact that incorporation of a CO enriched solution does not modify films transparency represents a favorable aspect regarding potential applications of these materials as food packaging.

\subsection{Diffusion assays}

Food packages with antimicrobial capacity require that active agents could migrate from polymeric matrix towards the product surface. Regarding the driving force for migration, the concentration gradient of the antimicrobial agent is the main contributor. Besides diffusion capability, CO should be released in a controlled way. This additional functionality not only depends on active agent characteristics, but also the matrix nature. Concerning active principle; size, shape and polarity are the most important factors that influence antimicrobial agent diffusion. On the other hand, chemical nature of the polymeric matrix, as well as its morphology also affect the controlled release (Pranoto et al., 2005; Cagri, Ustunol, \& Ryser, 2001). Furthermore, diffusion processes depends on the medium properties towards active agent migrates (polarity, $\mathrm{pH}$, state of aggregation, density, viscosity, etc.) and the physical conditions (temperature, pressure, relative humidity, etc).

Particularly, in this work oligochitosan diffusion from the active film to a liquid simulant medium was evaluated at three $\mathrm{pH}$ values. It should be noted that the assayed acidity conditions were chosen because they are typical of a wide variety of food products.

Fig. 4 shows experimental and fitted data corresponding to $\mathrm{CO}$ diffusion assays at the three $\mathrm{pHs}$ tested. As it can be observed, chitosan derivative was able to migrate from the starch matrix to the assayed media in the three $\mathrm{pH}$ conditions. The fastest diffusion rate was achieved during the first $30 \mathrm{~min}$, releasing more than $50 \%$ of the oligomer in the three studied simulant media. Furthermore, $\mathrm{CO}$ diffusion rate in the initial stage was increased with a decrease in $\mathrm{pH}$. After the first hour, CO concentration continued increasing slowly over the studied time. Moreover, it was evidenced that $\mathrm{CO}$ diffusion was slightly improved by reducing the $\mathrm{pH}$ medium in accordance to the affinity of chitosan derivatives with acid surroundings. In this sense, Amorim et al. (2016) stressed that the good dispersion of chitosan in acid solutions is 


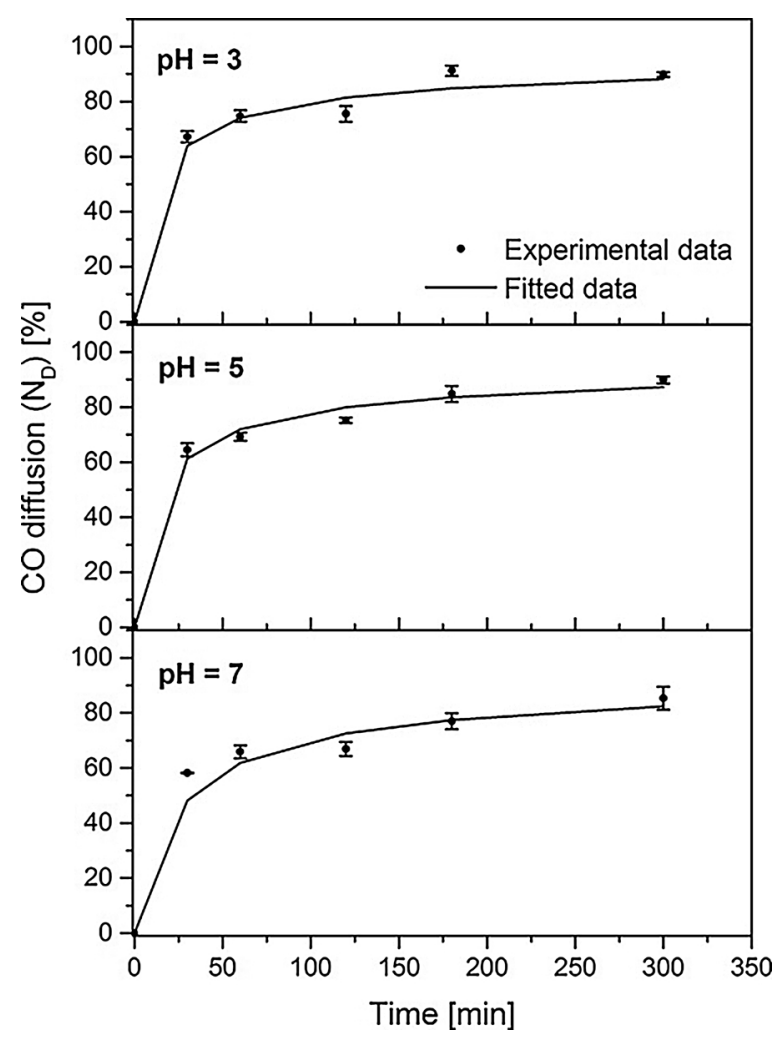

Fig. 4. Experimental and fitted data corresponding to chitosan oligomer (CO) diffusion assays from active film to simulant media at three pHs.

attributed to the protonation of amino groups, conferring positive electrical charges to the polysaccharide chains.

A mathematical model was developed in order to predict the $\mathrm{CO}$ diffusion process from the active film to a liquid simulant medium. Fig. 4 shows the fitting curves for the three assayed systems. Even though several simplifying assumptions were considered, a satisfactory fitting was achieved for $\mathrm{pH} 3\left(\mathrm{r}^{2}=0.93\right)$ and $\mathrm{pH} 5\left(\mathrm{r}^{2}=0.89\right)$. Concerning to $\mathrm{pH} 7$, the correlation coefficient was lower than 0.85 meaning that the proposed model did not properly fit the experimental data. In order to improve the model prediction, several factors should be considered in its expression. In this sense, model would be more accurate if there are included terms associated to: i) dissolution and swelling of TPS matrix, ii) dependence of diffusivity with position and $\mathrm{pH}$, iii) possible interactions between $\mathrm{CO}$ and TPS; iv) CO three-dimensional diffusion, and v) boundary effects. In a previous work, it was determined that water solubility of the TPS matrix used in this study was $21 \%$, at $\mathrm{pH}=7$ and $25{ }^{\circ} \mathrm{C}$ (Ninago et al., 2015). The CO diffusion in the aqueous neutral medium was estimated considering TPS solubility, obtaining a value of around $66 \%$ at the maximum assayed time. TPS dissolution during diffusion assays in aqueous simulant media could explain the low regression coefficients obtained by the mathematical fit of the experimental data. From the proposed simplified model, it was evidenced that diffusion coefficient depends on the medium acidity. Considering a constant diffusivity during CO migration, values of $2.49,2.13$ and $1.10 \times 10^{-7} \mathrm{~cm}^{2} \mathrm{~s}^{-1}$ were estimated for pH 3, 5 and 7, respectively.

\subsection{Antimicrobial capacity}

It is well known that chitosan and its derivatives are good inhibitors of molds and yeasts (Aider, 2010). Dutta, Tripathi, Mehrotra, and Dutta, (2009) stressed that chitosan antimicrobial activity depends not only on intrinsic factors but also to extrinsic ones. Among the polysaccharide intrinsic features, the most relevant are its molecular weight, degree of polymerization, and deacetylation level. Thus, Dutta et al. (2009) reported that chitosan oligomers with molecular weight lower than $10,000 \mathrm{~g} \mathrm{~mol}^{-1}$ and degree of polymerization of at least 7 have greater antimicrobial activity than native polysaccharide. This claim could be attributed to the higher aqueous solubility of chitosan oligomers compared to the solubility of the high molecular weight derivatives, favoring the reaction between the oligomers and the active sites of the microorganisms (Aider, 2010). Concerning to deacetylation degree, chitosan derivatives with low proportion of acetylated amino groups present higher antimicrobial capacity. Main extrinsic factors which limit chitosan active functionality are target microorganisms and media conditions ( $\mathrm{pH}$, ionic strength, and reactive solutes). It is well known that chitosan antimicrobial activity is enhanced by the presence of acid media due to low $\mathrm{pH}$ values induce a 'hurdle effect', exerting an acid stress on the microorganisms (Aider, 2010; Rhoades \& Rastall, 2000).

Microbiological assays were performed to evaluate the active capacity of $\mathrm{CO}$ to inhibit molds and yeasts growth in strawberries, ricotta, and flavored breads. Two different methodologies were compared: active packaging and spraying technique. Total counts of molds and yeasts of all assayed samples are shown in Fig. 5. Concerning to molds, active packaging and spraying method reduced significantly the growth of these microorganisms in all studied foods, compared to the corresponding control samples. Particularly, spraying method with $\mathrm{CO}$ enriched solution led to a reduction in molds growth of 70, 42, and $33 \%$ for strawberries, ricotta, and flavored breads, respectively. This antifungal effect resulted more pronounced when sachets based on the active film were used. In this regard, molds growth in packed strawberries, ricotta, and breads was $4,1.75$, and 2 times lower than the sprayed ones, respectively.

Total yeasts count of all control samples and food products treated with the spraying method was not significantly different between them. However, packaging of these foods using active sachets improved their protection against these microorganisms. In the case of strawberries and ricotta, the reduction of yeasts growth was $\sim 58 \%$; meanwhile for flavored breads was $86 \%$, in comparison to the corresponding control samples.

Even though, the observed tendency resulted similar among the three food products, the total counts of molds and yeasts were different. Flavored breads were the least susceptible product to the microbial development, while strawberries and ricotta presented the highest molds and yeasts growth, respectively. These differences could be related to the inherent characteristics of each product. As it was expected, foods with high water activity were more prone to microbial contamination.

Results derived from antimicrobial assays revealed that $\mathrm{CO}$ incorporation within material packaging is more effective than spraying technique to extend the food shelf-life. This experimental observation is explained by two possible phenomena:

i) active compound concentration in the spraying solution could be reduced by side reactions with some food components when the agent gets in contact with the product; ii) active agent could quickly migrate off food surface, turning unprotected the region of the product which is in contact with the media. On the other hand, the use of active packages assures a controlled release of the antimicrobial compound, favoring the protection of the food surface for a longer time. According to Hanušová, Dobiáš, and Klaudisová (2009), direct incorporation of active agents into food results in an immediate but short-term reduction of bacterial populations, while the antimicrobial films can maintain their activity for a longer period of time.

\section{Conclusions}

A prototype package was obtained at laboratory scale from active biodegradable films based on thermoplastic corn starch (TPS) and chitosan oligomer (CO). A non-commercial $\mathrm{CO}$ was obtained at 

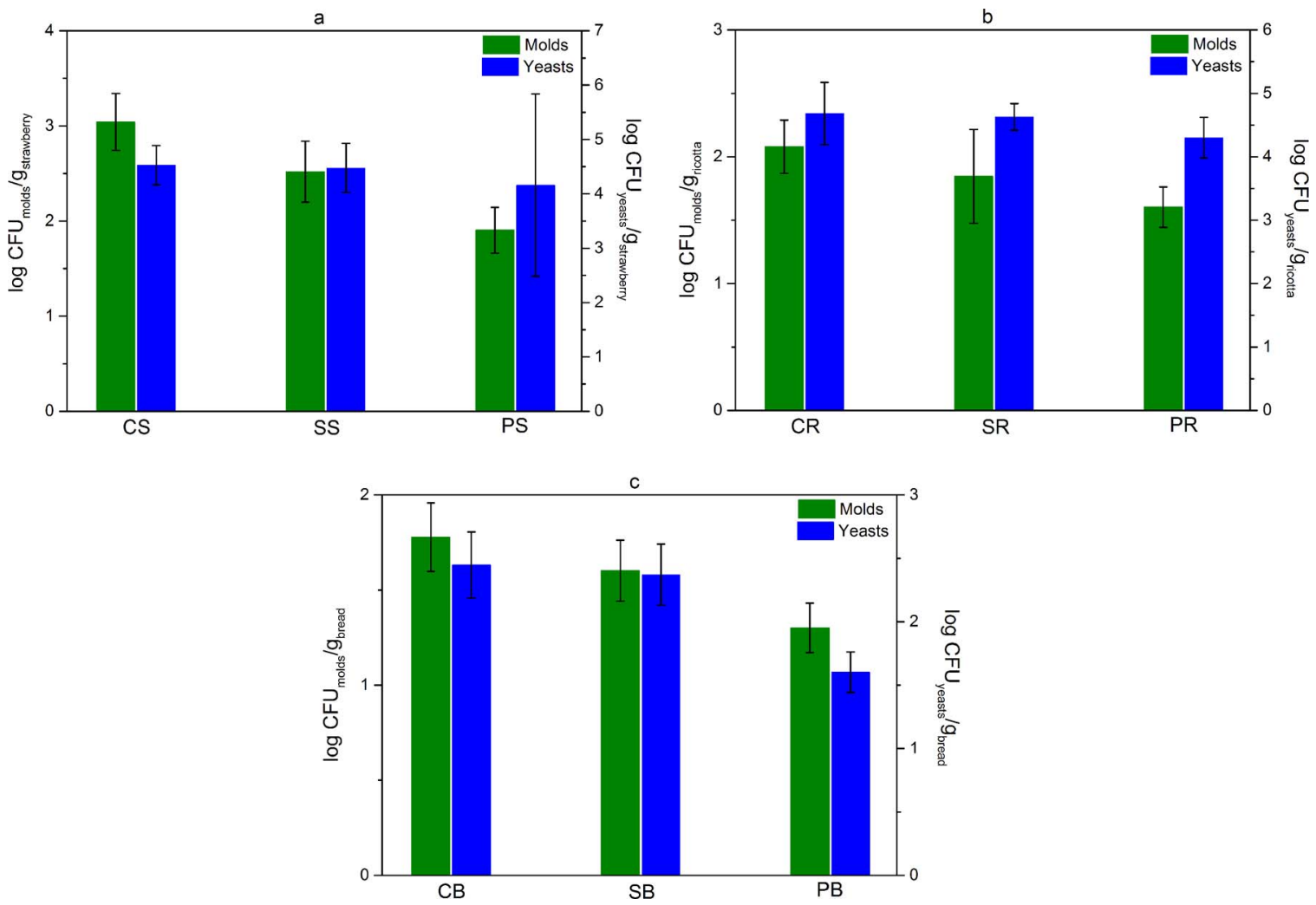

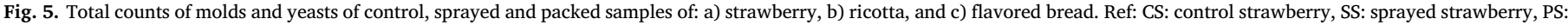
packed strawberry, CR: control ricotta, SR: sprayed ricotta, PR: packed ricotta, CB: control bread, SB: sprayed bread, PB: packed bread.

laboratory scale from chitosan, a polysaccharide derived from shrimp shells wastes, and incorporated as active compound to TPS films. It was demonstrated that the antimicrobial agent migrated from active films to aqueous simulant media due to intrinsic CO characteristics (low molecular weight and degree of polymerization), matrix nature and microstructure, and physicochemical properties of the media. Moreover, CO diffusion was not conditioned by the acidity of the simulant media in the $\mathrm{pH}$ studied range, highlighting the potentiality of using this antimicrobial agent for a wide variety of foods. Thanks to the development of a simplified mathematical model, it could be possible to predict CO diffusion behavior for different film thickness values, CO concentrations, and simulant media. Sachet type packages demonstrated to have a notable antimicrobial capability against molds and yeasts. Comparing the $\mathrm{CO}$ protective action when this agent was in solution (spraying method) and when it was incorporated to the packaging material, a higher antimicrobial effect was achieved using active sachets.

Results obtained in this work demonstrated the feasibility of using chitosan oligomers as antimicrobial agent in thermoplastic starch films to develop active food packaging.

\section{References}

Aider, M. (2010). Chitosan application for active bio-based films production and potential in the food industry: Review. LWT - Food Science and Technology, 43(6), 837-842. Alvarado-González, J. S., Chanona-Perez, J. J., Welti-Chanes, J. S., Calderon-Dominguez, G., Arzate-Vazquez, I., Pacheco-Alcalá, S. U., et al. (2012). Propiedades ópticas microestructurales, funcionales y nanomecánicas de películas comestibles de gel de Aloe vera/goma gelano. Revista Mexicana de Ingeniería Química, 11(2), 193-210.

Amorim, M. L., Ferreira, G. M. D., Soares de S, L., Soares dosS, W. A., Ramos, A. M. Coimbra dos R, J. S., et al. (2016). Physicochemical aspects of chitosan dispersibility in acidic aqueous media: Effects of the food acid counter-anion. Food Biophysics, 11(4), 388-399.

Appendini, P., \& Hotchkiss, J. H. (2002). Review of antimicrobial food packaging. Innovative Food Science \& Emerging Technologies, 3(2), 113-126.

Barzegar, H., Azizi, M. H., Barzegar, M., \& Hamidi-Esfahani, Z. (2014). Effect of potassium sorbate on antimicrobial and physical properties of starch?clay nanocomposite films. Carbohydrate Polymers, 110, 26-31.

Brück, W. M., Slater, J. W., \& Carney, B. F. (2010). Chitin and chitosan from marine organisms. In K. Se-Kwon (Ed.). Chitin, chitosan, oligosaccharides and their derivatives: Biological activities and applications (pp. 11-23). Boca Raton: CRC Press.

Cagri, A., Ustunol, Z., \& Ryser, E. (2001). Antimicrobial mechanical, and moisture barrier properties of low $\mathrm{pH}$ whey protein-based edible films containing $\mathrm{p}$-Aminobenzoic or sorbic acids. Journal of Food Science, 66(6), 865-870.

Cano, A., Cháfer, M., Chiralt, A., \& González-Martínez, C. (2015). Physical and antimicrobial properties of starch-PVA blend films as affected by the incorporation of natural antimicrobial agents. Foods, 5(1), 3.

Castillo, L. A., López, O. V., López, C., Zaritzky, N. E., García, M. A., Barbosa, S. E., et al. (2013). Thermoplastic starch films reinforced with talc nanoparticles. Carbohydrate Polymers, 95(2), 664-674.

Castillo, L. A., López, O. V., Ghilardi, J., Villar, M. A., Barbosa, S. E., \& García, M. A. (2015). Thermoplastic starch/talc bionanocomposites. Influence of particle morphology on final properties. Food Hydrocolloids, 51, 432-440.

Corazzari, I., Nisticò, R., Turci, F., Faga, M. G., Franzoso, F., Tabasso, S., et al. (2015) Advanced physico-chemical characterization of chitosan by means of TGA coupled on-line with FTIR and GCMS: Thermal degradation and water adsorption capacity. Polymer Degradation and Stability, 112, 1-9.

Corrales, M., Fernández, A., \& Han, J. H. (2014). Antimicrobial packaging systems. In Han, \& H. Jung (Eds.). Innovations in food packaging (pp. 133-170). (2nd ed.). San Diego: Academic Press.

Costa-Júnior, E. S., Barbosa-Stancioli, E. F., Mansur, A. A. P., Vasconcelos, W. L., \& Mansur, H. S. (2009). Preparation and characterization of chitosan/poly(vinyl alcohol) chemically crosslinked blends for biomedical applications. Carbohydrate Polymers, 76(3), 472-481.

Dutta, P. K., Tripathi, S., Mehrotra, G. K., \& Dutta, J. (2009). Perspectives for chitosan based antimicrobial films in food applications. Food Chemistry, 114(4), 1173-1182.

Flores, S., Haedo, A. S., Campos, C., \& Gerschenson, L. (2007). Antimicrobial performance of potassium sorbate supported in tapioca starch edible films. European Food Research and Technology, 225(3-4), 375-384.

Goy, R. C., de Britto, D., \& Assis, O. B. G. (2009). A review of the antimicrobial activity of chitosan. Polímeros, 19(3), 241-247.

Goy, R. C., Morais, S. T. B., \& Assis, O. B. G. (2016). Evaluation of the antimicrobial activity of chitosan and its quaternized derivative on E. coli and S. aureus growth. Revista Brasileira de Farmacognosia, 26(1), 122-127.

Guerrero, P., Beatty, E., Kerry, J., \& De La Caba, K. (2012). Extrusion of soy protein with gelatin and sugars at low moisture content. Journal of Food Engineering, 110(1), 53-59.

Hanušová, K., Dobiáš, J., \& Klaudisová, K. (2009). Effect of packaging films releasing antimicrobial agents on stability of food products. Czech Journal of Food Sciences, 27 347-349.

Hussain, I., Singh, T., \& Chittenden, C. (2012). Preparation of chitosan oligomers and 
characterization: Their antifungal activities and decay resistance. Holzforschung, 66(1), 119-125.

Juneja, V. K., Dwivedi, H. P., \& Yan, X. (2012). Novel natural food antimicrobials? Annual Review of Food Science and Technology, 3(1), 381-403.

Khanmohammadi, M., Elmizadeh, H., \& Ghasemi, K. (2015). Investigation of size and morphology of chitosan nanoparticles used in drug delivery system employing chemometric technique. Iranian Journal of Pharmaceutical Research: IJPR, 14(3), 665.

Kuorwel, K. K., Cran, M. J., Sonneveld, K., Miltz, J., \& Bigger, S. W. (2011). Antimicrobial activity of natural agents coated on starch-based films against staphylococcus aureus? Journal of Food Science, 76(8), M531-M537.

Kuorwel, K. K., Cran, M. J., Sonneveld, K., Miltz, J., \& Bigger, S. W. (2013). Migration of antimicrobial agents from starch-based films into a food simulant. LWT - Food Science and Technology, 50(2), 432-438.

López, O. V., García, M. A., \& Zaritzky, N. E. (2008). Film forming capacity of chemically modified corn starches. Carbohydrate Polymers, 73(4), 573-581.

López, O. V., Giannuzzi, L., Zaritzky, N. E., \& García, M. A. (2013). Potassium sorbate controlled release from corn starch films. Materials Science and Engineering: C, 33(3), $1583-1591$.

López, O. V., Garcia, M. A., Villar, M. A., Gentili, A., Rodriguez, M. S., \& Albertengo, L. (2014). Thermo-compression of biodegradable thermoplastic corn starch films containing chitin and chitosan. LWT - Food Science and Technology, 57(1), 106-115.

López, O. V., Castillo, L. A., García, M. A., Villar, M. A., \& Barbosa, S. E. (2015). Food packaging bags based on thermoplastic corn starch reinforced with talc nanoparticles. Food Hydrocolloids, 43, 18-24.

Leceta, I., Guerrero, P., \& de la Caba, K. (2013). Functional properties of chitosan-based films. Carbohydrate Polymers, 93(1), 339-346.

Lucera, A., Costa, C., Conte, A., \& Del Nobile, M. A. (2012). Food applications of natural antimicrobial compounds. Frontiers in Microbiology, 3, 287.

Marroquin, J. B., Rhee, K. Y., \& Park, S. J. (2013). Chitosan nanocomposite films: Enhanced electrical conductivity, thermal stability, and mechanical properties. Carbohydrate Polymers, 92(2), 1783-1791.

Mehdizadeh, T., Tajik, H., Razavi Rohani, S. M., \& Oromiehie, A. R. (2012). Antibacterial, antioxidant and optical properties of edible starch-chitosan composite film containing Thymus kotschyanus essential oil. Veterinary Research Forum, 3(3), 167-173.

Mohamed, R. R., Elella, M. H. A., \& Sabaa, M. W. (2015). Synthesis: Characterization and applications of $\mathrm{N}$ - quaternized chitosan/poly(vinyl alcohol) hydrogels. International Journal of Biological Macromolecules, 80, 149-161.

Moreno, O., Atarés, L., \& Chiralt, A. (2015). Effect of the incorporation of antimicrobial/ antioxidant proteins on the properties of potato starch films. Carbohydrate Polymers, 133, 353-364.

Moussout, H., Ahlafi, H., Aazza, M., \& Bourakhouadar, M. (2016). Kinetics and mechanism of the thermal degradation of biopolymers chitin and chitosan using thermogravimetric analysis. Polymer Degradation and Stability, 130, 1-9.

Nallamuthu, I., Devi, A., \& Khanum, F. (2015). Chlorogenic acid loaded chitosan nanoparticles with sustained release property, retained antioxidant activity and enhanced bioavailability. Asian Journal of Pharmaceutical Sciences, 10(3), 203-211.

Ninago, M. D., López, O. V., Lencina, M. M. S., García, M. A., Andreucetti, N. A., Ciolino, A. E., et al. (2015). Enhancement of thermoplastic starch final properties by blending with poly(E-caprolactone). Carbohydrate Polymers, 134, 205-212.

No, H. K., Meyers, S. P., Prinyawiwatkul, W., \& Xu, Z. (2007). Applications of chitosan for improvement of quality and shelf life of foods: A review. Journal of Food Science, 72(5), R87-R100.

Pathare, P. B., Opara, U. L., \& Al-Said, F. A. (2013). Colour measurement and analysis in fresh and processed foods: A review. Food and Bioprocess Technology, 6(1), 36-60.
Pillai, S. K., \& Ray, S. S. (2012). Chitosan-based nanocomposites. In J. J. Maya, \& S. Thomas (Vol. Eds.), Natural polymers volume 2: Nanocomposites: Vol. 2, (pp. 33-68). The Royal Society of Chemistry.

Pranoto, Y., Rakshit, S. K., \& Salokhe, V. M. (2005). Enhancing antimicrobial activity of chitosan films by incorporating garlic oil, potassium sorbate and nisin. LWT - Food Science and Technology, 38(8), 859-865.

Rabea, E. I., Badawy, M. E.-T., Stevens, C. V., Smagghe, G., \& Steurbaut, W. (2003). Chitosan as antimicrobial agent: Applications and mode of action? Biomacromolecules, 4(6), 1457-1465.

Rawat, P., \& Singh, R. N. (2015). Eco-friendly synthesis, spectral and computational study of pyrrole-2-carboxaldehyde salicylhydrazone (PCSH) for its application. Journal of Molecular Structure, 1100, 105-115.

Rhoades, J., \& Rastall, B. (2000). Chitosan as an antimicrobial agent. Food Science Technology International, 32-33.

Romainor, A. N. B., Chin, S. F., Pang, S. C., \& Bilung, L. M. (2014). Preparation and characterization of chitosan nanoparticles-doped cellulose films with antimicrobial property. Journal of Nanomaterials, 2014, 130.

Salleh, E., Muhammad, I. I., \& Pahlawi, Q. A. (2014). Spectrum activity and lauric acid release behaviour of antimicrobial starch-based film. Procedia Chemistry, 9, 11-22.

Shao, J., Yang, Y., \& Zhong, Q. (2003). Studies on preparation of oligoglucosamine by oxidative degradation under microwave irradiation. Polymer Degradation and Stability, 82(3), 395-398.

Srinivasa, P. C., \& Tharanathan, R. N. (2007). Chitin/Chitosan — safe, ecofriendly packaging materials with multiple potential uses. Food Reviews International, 23(1), 53-72.

Touch, V., Hayakawa, S., \& Commins, T. (2009). Natural antimicrobial proteins: A review of current challenges and solutions for food applications. Asian Journal of Food and Agro-Industry, 2(1), 1-16.

Tripathi, S., Mehrotra, G. K., \& Dutta, P. K. (2008). Chitosan based antimicrobial films for food packaging applications. E-Polymers, 8(1), 93.

Wu, T., \& Zivanovic, S. (2008). Determination of the degree of acetylation (DA) of chitin and chitosan by an improved first derivative UV method. Carbohydrate Polymers, 73(2), 248-253.

Yasir, S. B. M., Sutton, K., Newberry, M., Andrews, N., \& Gerrard, J. (2007). The impact of Maillard cross-linking on soy proteins and tofu texture. Food Chemistry, 104(4), 1502-1508.

Zainal, Z., Hui, L. K., Hussein, M. Z., Abdullah, A. H., \& Hamadneh, I. M. K. R. (2009). Characterization of TiO2-Chitosan/Glass photocatalyst for the removal of a monoazo dye via photodegradation-adsorption process. Journal of Hazardous Materials, 164(1), 138-145.

Zhang, L., Pornpattananangkul, D., Hu, C.-M., \& Huang, C.-M. (2010). Development of nanoparticles for antimicrobial drug delivery? Current Medicinal Chemistry, 17(6), 585-594.

Zheng, X., Yin, Y., Jiang, W., Xing, L., \& Pu, J. (2015). Synthesis and characterization of low molecular weight chitosan. BioResources, 10(2), 2338-2349.

Ziegler-Borowska, M., Chełminiak, D., \& Kaczmarek, H. (2015). Thermal stability of magnetic nanoparticles coated by blends of modified chitosan and poly(quaternary ammonium) salt. Journal of Thermal Analysis and Calorimetry, 119(1), 499-506.

Zuñiga, A., Debbaudt, A., Albertengo, L., \& Rodríguez, M. S. (2010). Synthesis and characterization of N-propyl-N-methylene phosphonic chitosan derivative. Carbohydrate Polymers, 79(2), 475-480.

van den Broek, L. A. M., Knoop, R. J. I., Kappen, F. H. J., \& Boeriu, C. G. (2015). Chitosan films and blends for packaging material. Carbohydrate Polymers, 116, 237-242. 